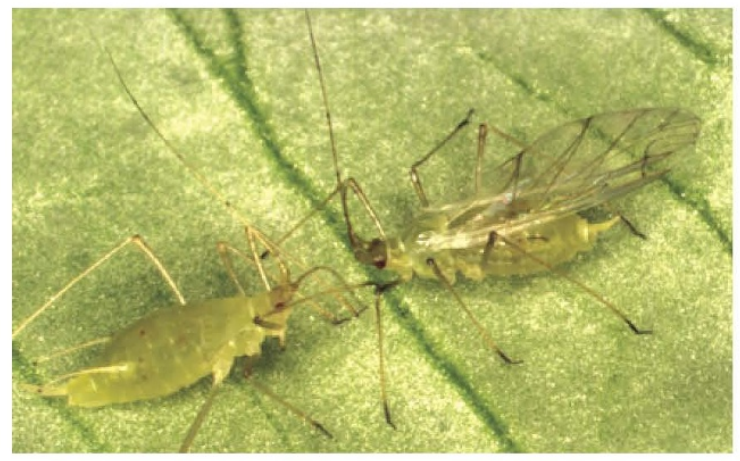

Image courtesy of David Stern, University of Princeton.

\title{
Aphids: a winged response
}

Variation in a gene that underlies a winged versus wingless polymorphism in male aphids is also linked to plasticity of the same trait in females, a new study shows. This striking finding indicates that organisms in which both genetic and environmental cues can trigger such morphological variation might be useful model systems in which to address the enduring mystery of phenotypic plasticity.

For a long time we have known of the ubiquitous importance of phenotypic plasticity - the ability to alter genetic developmental programmes in response to environmental variation - as a way of dealing with predictably variable environments. Which makes it all the more disappointing that the underlying mechanisms remain elusive.

One key (previously unanswered) question that Christian Braendle and colleagues address in their new study relates to phenotypically plastic traits that mimic genetically controlled polymorphisms. Are the genes that underlie genetically controlled polymorphisms involved in mediating phenotypically plastic traits?

The authors studied parthenogenetic female clones of the pea aphid, Acyrthosiphon pisum, that produce offspring that develop wings to varying frequencies. This adaptive 'polyphenism', which causes the frequency of winged offspring to increase under adverse environmental conditions, mimics the wing polymorphism in male pea aphids that is controlled by the biallelic, X-linked, aphicarus (api) locus.

In response to crowding and starvation, clones that are homozygous for the winged api allele $\left(a p i^{w}\right)$ are significantly less likely to produce winged female offspring than clones that are homozygous for the wingless allele (api $\left.i^{w \prime}\right)$ and than heterozygous clones $\left(a p i^{w} / a p i^{w l}\right)$. This pattern was replicated in subsequent experiments that more closely mimicked natural conditions for these clones over a longer period of time.

The inverse relationship of the female polyphenism and the male polymorphism is intriguingly counterintuitive and, as the authors highlight, requires further investigation. Nonetheless, this pattern could be explained as the pleiotropic effects of a single gene: a hypothesis that can only be addressed with more extensive characterization of api.

Regardless of the results of such follow-up studies, the authors' approach provides a model for identifying genes that might be involved in mediating plastic developmental responses to environmental cues, which is a key intermediate goal in the search for a general explanation for phenotypic plasticity.

Nick Campbell, NPG Executive Editor, Heredity

(3) References and links

ORIGINAL RESEARCH PAPER Braendle, C. et al. Genetic variation for an aphid wing polyphenism is genetically linked to a naturally occurring wing polymorphism. Proc. R. Soc. B 30 March 2005 (doi:10.1098/rspb.2004.2995)

FURTHER READING Braendle, C.et al. Genetic mapping of aphicanus - a sex-linked locus controlling a wing polymonhism in the pea aphid (Acyrthosiphon pisum). Heredity 94, 435-442 (2005)

WEB SITE

David Stern's laboratory: http://www.princeton. edu/\%7Edstern/index.htm 